\title{
Man, Woman, Engineer, Psychologist: Mixed Messages in Research Design
}

\author{
Francis J. Hopcroft, P.E., LSP, Barbara A. Karanian, Ph.D. \\ Wentworth Institute of Technology \\ Boston, MA 02115
}

\begin{abstract}
Several years ago an off-hand remark by Professor Karanian, a Psychologist, during a casual conversation with Professor Hopcroft, an Engineer, led to a decision to jointly research the way men and women respond to technical presentations with which they disagree. A working hypothesis was developed, after some discussion about the meaning of the words in the title, and a conceptual plan of study was defined. The plan of study fundamentally involved observing men and women responding to presentations at technical conferences and recording those observations in a standardized format. This paper addresses the communication and procedural difficulties that arose as the two professionals, each competent in their own area of expertise, tried to meld two very different approaches to research into a successful research effort.
\end{abstract}

\section{Introduction}

Teaching engineering students on a campus that encourages collaboration across departments opened a door to understanding the different ways engineers and psychologists think and behave. Working with professors across disciplines and with industry consultants expanded that understanding further. An attempt at a research collaboration between an engineering professor and a psychology professor highlighted these differences. Questions emerged, however, that strained the professors' ability to agree on the research design and complete the study.

They responded to the challenges during the collaboration by changing the research design, redefining the variables, and shifting the procedure for data collection. Despite these creative efforts, and extensive communication between the two professors, the research study was not completed. Both expressed some frustration with the situation and raised questions about mixedmessages in research design.

Understanding of their differences begins in a discussion of methodology, incorporates communication, and ends in culture. The primary objective of the work shifted from successfully completing the original research study to the much more difficult topic of interdisciplinary and cross-gender collaboration. The new focus is designed to facilitate the formulation of a plan that examines key issues that impact successful collaboration. The fact that gender is also a variable presents intriguing and only sometimes predictable influences on the working relationship. This paper provides an informal and descriptive framework for conceptualizing the collaborative research effort by considering expectations for successful project completion, and implications for further study.

\section{Overview of Themes}

This paper began with some hunches about the effects that profession has on academic or industry collaboration. Consistent attempts to develop appropriate research instruments

"Proceedings of the 2002 American Society for Engineering Education Annual Conference \& Exposition Copyright (C) 2002, American Society for Engineering Education” 
suggested that different definitions of research methodology terms were being used. While one demanded, "What are the hypothesis?" the other responded, "Hypothesis? How can we talk about the hypothesis without the data?"

Based on empirical observations of other research and anecdotal data identified during the initial research effort, three distinct themes evolved to provide a framework for discussion: 1) methodology, 2) communication, and 3) culture.

\section{A. Methodology}

The complex structure of the mind is not the subject of this paper. Relevant here are key ideas concerning thinking across professions. For both the engineer and the psychologist new models of understanding methodology and innovative approaches to research have forever changed the nature of their research. Prior researchers ${ }^{[1,2,3]}$ revolutionized thinking about thinking. New models of thought recognize the importance of innovation, creativity, and culture. That input influenced the new direction in the current collaboration and suggested that research also needs to be defined by innovation, creativity, and culture.

A differentiation between the research engineer and the practicing engineer in the way that they approach research may be significant. Research engineers often approach an issue from several viewpoints and may begin their research efforts in much the same way as a chemist or even a psychologist. The practicing engineer, in discussing work with other engineers typically agrees on one predictable methodology. The approach usually varies only slightly from the following steps: 1) problem identification, 2) definition and evaluation of alternatives, 3) selection of the optimal solution, 4) and implementation of the selected alternative.

In contrast, psychologists often use varied approaches to research based on whether lab or field research is conducted. Very different systems of thinking frame the research in psychology. While not universally accepted, four foundational systems are frequently cited in psychology: 1) Psychoanalytic, 2) Learning, 3) Cognitive, and 4) Humanistic. These may be combined or stand alone to provide different theoretical frameworks for research design. While it is not possible to completely define each system in one sentence, the definitions below are used in this discussion of methodology.

Past, deeply internalized conflicts or other 'unknowing' ways of thinking and feeling are the basis for the Psychoanalytic system ${ }^{[4]}$. For example, the word "imagine" was used on the original research instrument. While the psychologist's intention was to use the word to create a projective and diagnostic measurement tool, the engineer had difficulty with the concept of a projective measure.

Learning or Behaviorism is based on stimulus-response behavior ${ }^{[5]}$. Instead of using the current research collaboration as an example, consider the entrance of the 'abrasive dean' into a faculty meeting. The abrasive dean creates a punishing, non-nurturing, and uncomfortable environment for faculty. The faculty automatically respond anxiously. 
The Cognitive system is based on intellectual development and information processing. For example, the psychologist remembered the details about one conversation concerning research procedure differently than the engineer. This is a good illustration of the cognitive system's computational-model of the different ways memory stores information for females and males ${ }^{[1]}$.

The Humanistic system is based on the premise that everyone has creative potential and that no one is born 'bad.' In the present collaboration, both the psychologist and the engineer are competent and confident in their specialized fields. The engineer easily recognized and accepted the ability of the psychologist in the areas of research and human behavior, and the psychologist easily recognized and accepted the ability of the engineer to develop plans for generating data, evaluating data and to manage a project. Although both use dramatically different approaches to achieve similar outcomes, each recognize creative potential in the other ${ }^{[4]}$.

\section{B. Communication}

"It was a dark and gloomy night," began the message with classic bravado. " The psychologist pondered whether the perception of gloom was a function of the value of darkness and whether that value judgment was properly asserted on the basis of the intellectual derivation of nonphysical phenomena suggested by the assertion. The engineer pondered how the relative value of dark versus light had been determined, whether the function of time had been properly considered, and what units of gloom had been used to formulate the assertion." This whimsical E-mail response was sent from the engineer to the psychologist when there were mixed messages in their research design. Brief in length, the letter had a significant impact on their working relationship. It became a memorable turning point in their research.

The creative E-mail communication was the engineer's attempt to define differences in thinking between the two professionals. The humor was a successful beginning in redirecting the focus of the research. The issues that emerged from the communication facilitated a change in three areas. First, instead of continuing with the original research premise it became more relevant and timely to consider the difficulties encountered during the collaboration. Second, differences in language and communication, used both in research design and collaboration presented a problem. Finally, the engineer and the psychologist began to wonder whether gender was a variable.

One area of confusion was deciphering verbal communication cues between professionals. Consideration of anecdotal classroom and industry data suggested that engineers do not understand the words psychologists use. Further, engineers indicate that they are not only unclear about the definitions of words, they are also unfamiliar with the underlying meaning of the language psychologists sometimes use. Expanding on an example from the methodology discussion, this occurred when the word "imagine" was used on the draft for a portion of the original research instrument. The psychologist's intention was to use the word "imagine" to create a projective measurement tool. The engineer had difficulty in understanding that particular use of the word, the concept of projective measurement, and any use of imagination to achieve a research goal. While various interpretations of Psycholinguistic research is relevant here, particularly useful is evidence of a 'speaker vs. hearer distinction that exists' when attempting to understand the meaning of the language ${ }^{[6]}$.

"Proceedings of the 2002 American Society for Engineering Education Annual Conference \& Exposition Copyright $\odot$ 2002, American Society for Engineering Education” 
Gender emerged as a variable with respect to the development of the research design and in creating the procedure for the collection of data. The initial disagreement concerned the significance of the gender make-up of the study sample. Whether the percentage of males to females in the study group was proportional to the population observed was perceived as irrelevant by the engineer. Additionally, the importance of considering the gender of data collector was not originally recognized as a significant variable by the engineer. While these issues overlap the earlier discussion of methodology, the communication impasse immobilized the research.

In explaining the pattern of gender differences in expectation, researchers ${ }^{[7,8]}$ argue that segregation by sex is common among children and adults in our cultures, and that norms are often well established for sex-segregated sports, professions, and games. An intertwining of developmental and group theory shapes the distinction between the way men and women "ought" to behave vs. what they "do" in same-sex and mixed-sex groups. We may be less clear about the appropriate norms for the mixed-sex groups and modify our behavior to fit the type of interaction we "expect" from the opposite sex ${ }^{[8]}$.

Implications for communication in work relationships emerge. Researchers ${ }^{[9,10,11,12]}$ learned that assertive women in communication, although considered more competent than non-assertive women by both male and female respondents, were not responded to favorably by men. Men not only liked and trusted the non-assertive, self-deprecating women they were more likely to be influenced or persuaded to change their mind by the women they considered less competent. In other words, the men liked and were persuaded by the women who acted less clear, competent, and assertive in communication. The women, on the other hand, liked and were persuaded by both the men and the women who demonstrated clear, competent, and assertive communication. Although a gender dichotomy in persuasive speech was not supported by the anecdotal experience in the current research effort, it led to conversations about the potential for these influences to impact the work.

Research suggesting that when men communicate together, the trend is to discuss a limited number of topics, is informative in the discussion of cues in communication. In contrast, when women communicate together, the trend is to discuss a multitude of topics, and multi-levels of the same topic. Many research discussions ${ }^{[9,10,12]}$ consider the relational quality of discussions among men and women at work. Leadership implications are particularly intriguing. Rich Priori, CEO and president of Duke Power and Electric explained that all male management discussions at the top of the organization were more effective when women participated. The all male discussi seemed to focus on one topic with a single voice. He was so convinced that the woman's voice added an important depth dimension that when he observed all male meetings he encouraged the addition of women ${ }^{[13]}$.

Results of the engineer and psychologist's collaboration further supported their original research premise concerning the different ways men and women challenge technical presentations. Specifically, they hypothesize that men tend to challenge the methodology while women tend to challenge the implications of the data.

"Proceedings of the 2002 American Society for Engineering Education Annual Conference \& Exposition Copyright (C) 2002, American Society for Engineering Education” 


\section{Culture}

Culture is the collection of attitudes and actions that so dramatically defines a given group that it is taught to new members as the correct way to perceive, feel, think and act ${ }^{[14]}$ in relation to that group. Culture varies from setting to setting and is built upon an historical and often legendary foundation. The culture of an academic setting varies by size, geography, staff, faculty, students, norms, values and policies that encourage cross-professional collaboration.

Culture is also a function of both compatibility among colleagues and the personality of the organization. Individuals themselves are complex ${ }^{[15]}$. In the context of organizations with diverse needs, individuals contribute productively to the organization only if the individual is in balance personally and with the organization. The organization fulfills multi-level needs for balance and a sense of what is just ${ }^{[15]}$. During this collaboration, the researchers agreed on a dynamic motivational venture, and the academic organization provided a structure to accomplish the task. This culture and working environment provided a mechanism for looking at the collaborative difficulties. As a result, expansion of the original concept occurred and provided an engaging opportunity for professional growth.

Part of the concept of how engineer's and psychologist's work seems to be contained in their professional background and the ways they approach their work. Engineers generally tend to try to narrow the scope of what they are trying to accomplish. Psychologists tend to be more willing to explore the various ramifications of their work. During collaborative efforts, the engineer's need to achieve an end point may clash with the psychologist's need to achieve an evolving evaluation and refinement of the question.

When engineers and psychologist respond in automatic and reflexive ways, whether in the classroom or conducting research, they are engaging in "mindless thinking ${ }^{[16]}$." Both professionals were trapped by categories. The need for the creation of new categories, learned during the collaboration on the original research, became obvious fairly quickly. Creating new categories, and letting go of a rigid reliance on shared professional realities, constructs and ideas is the beginning of mindfulness. Being forced into that mindfulness helped to define the issues that had been interfering with effective collaboration. Labeling and relabeling as one masters the world, are processes, not natural, but necessary for productive work ${ }^{[16]}$.

\section{Implications from the Collaborations}

Conceptualization of the themes of methodology, communication, and culture set the stage for a different definition of successful collaboration. Specific areas of clarity that emerged concerning methods and ways of communication across professional and gender lines will facilitate future collaboration.

It happens in science that the most useful outcome is as much a function of circumstance and timing as it is a function of determination and design. Such was the case here. The concepts around which the original research effort was designed turned out to be as interesting as the examination of why a successful outcome has not yet been achieved.

"Proceedings of the 2002 American Society for Engineering Education Annual Conference \& Exposition Copyright $\odot$ 2002, American Society for Engineering Education" 
It was recognized that if the causes of failure could be clarified, the roots of success could be planted. Failure analysis is, in fact, a key tool in the practicing engineer's toolbox and perhaps a useful one for other professions to emulate. Interdisciplinary collaboration appears to be one of those places.

Clarity about an engineer/psychologist collaboration presents distinct advantages. First, heightened awareness about the nature of the profession is the basis for successful combined productivity. In addition, heightened awareness about the underlying thinking that drives the way interdisciplinary and cross gender collaborators approach a research project can minimize many areas of potential conflict. To the extent that the issues discussed in this presentation can enhance that understanding, both from a professional collaborative level and from a cross gender standpoint, the effort has been successful.

Second, the collaboration created an opportunity for an in-depth consideration of concepts that would never have been considered under normal circumstances. The engineering profession, not unlike the psychology profession, is fraught with jargon disguised as professional language. Professionals need to learn to minimize use of jargon and to understand the language of other professions. Similarly, men and women tend to communicate with each other in very different ways when they communicate with members of the opposite sex. Cross gender collaboration can be optimally successful when these differences are recognized and managed.

While the immediate efforts have not included specific research design elements or designed specific steps toward achievement of the original collaboration goals, it has succeeded in clarifying the difficulties of interdisciplinary and cross gender collaborations. Although these understandings are derived from anecdotal data and empirical observations of research, they will go a long way toward improving the success of future research efforts.

\section{Summary}

In summary, this presentation served three purposes. First, the mixed messages in research collaboration provided a mechanism for considering the interactive nature of the engineering and psychology profession. Second, recognition of distinct differences in methodology, communication, and attitude enhanced understanding and was as significant as the collaboration itself. Finally, the collaboration forced changes in the research focus and that required new ways of thinking.

\section{References}

[1] Pinker, How the Mind Works, Norton Press, 1997.

[2] Gould, S., The Mismeasure of Man, WW Norton, 1996.

[3] Gardner, H., The Disciplined Mind, Penguin, 2001.

[4] Fernald, D. The Hans Legacy, Lawrence Erlbaum, NJ, 1984.

[5] Hersey, P., Blanchard, K. Management of Organizational Behavior, Prentice, 1993.

[6] Chomsky, N., Logical Structure of Linguistic Theory, University of Chicago Press, 1985 .

[7] Karanian,B., Engineer's Response to Leadership: Does Gender Make a 
Difference? (unpublished document, 1991) Presented at ASEE/FIE 1992.

[8] Maccoby, E., "Gender Relationships." American Psychologist, 45(4), p.513-529.

[9] Carli, L., "Gender Differences in Interaction Style and Influence." Journal of Personality and Social Psychology, 1989, vol. 56, no. 4, p.565-576.

[10] Tannen, D., You Just Don't Understand: Men and Women in Conversation, Quill Press, 2001.

[11] Karanian,B., Gender and Leadership: Men and Women's Stories, dissertation, 1995.

[12] Levant, R., "Toward the Reconstruction of Masculinity." Journal of Family Psychology, 5, 1992.

[13] Priori, R., Interviewing a Leader Through Times of Change. Unpublished, 2000.

[14] Schein, E. Organizational Culture and Leadership, Jossey-Bass, 1988.

[15] Argyris, C. and Schon,D., Organizational Learning II, Addison-Wesley, 1996.

[16] Langer, E., Mindfulness. Addison-Wesley, 1989.

FRANCIS J. HOPCROFT: Associate Professor of Environmental Engineering, Wentworth Institute of Technology, Boston, MA., specializing in the areas of hazardous waste management, water and wastewater treatment, and in-situ bioremediation.

BARBARA A. KARANIAN: Professor of Social Science and Management, Wentworth Institute of Technology, Boston, MA. Research and teaching areas focus on the applied psychology areas of motivation and career path, leadership, and transformation. Works creatively with changing organizations. 